\title{
Legitimität von Wahlen in der Transformation
}

Als die „dritte Welle der Demokratisierung“ (Huntington 1991) vor etwas mehr als zwei Jahrzehnten das subsaharische Afrika erfasste, ${ }^{1}$ zweifelten damals nicht wenige an der Dauerhaftigkeit der politischen Liberalisierung des Kontinents (Huntington 1991; Joseph 1991; McFerson 1992). Zahlreiche zeitgenössische Studien der vergleichenden Politikwissenschaft zeigen heute, dass die politischen Reformen in vielen Ländern nachhaltiger gewesen sind als zunächst gedacht. ${ }^{2}$ Von der deutschen Öffentlichkeit wurde Afrikas politischer Wandel lange Zeit ausgeblendet. ${ }^{3}$ Auch heute findet er in den Medien selten Aufmerksamkeit. Für viele mag daher die Feststellung ungewohnt erscheinen, dass autokratische Herrschaftsmodelle in Afrika nicht mehr die Regel, sondern die Ausnahme sind. Länder ohne Mehrparteienwahlen findet man selbst in autokratisch regierten Ländern kaum. ${ }^{4}$

Die bisherige Bilanz der Demokratisierung ist dennoch durchwachsen. Afrikas Transitionsversuche sind mit mindestens zwei Einschränkungen versehen. Erstens haben sich die allermeisten afrikanischen Staaten trotz regelmäßig stattfindenden Wahlen nicht zu konsolidierten liberalen Demokratien entwickelt. In vielen Ländern werden Mehrparteienwahlen immer noch von Amtsinhabern manipuliert. Dies geschieht entweder durch direkte Manipulation des Parteienwettstreites selbst (beispielsweise durch die Besetzung von Wahlkommissionen mit politisch abhängigem Personal) oder durch eine indirekte Störung des politischen Wettbewerbs (beispielsweise durch die Fixierung der staatlichen Medien auf die derzeitigen Machthaber und deren Programm). Die Mehrzahl afrikanischer Regime werden daher als „,defekte Demokratien“ oder „hybride Regime“ klassifiziert (Erdmann 2002; Levitsky/Way 2011; Merkel u. a. 2003). Zweitens werden afrikanische Wahlen oftmals von schweren Gewaltausbrüchen überschattet. Die jüngsten Medienberichte von

1 In diesem Beitrag bezieht sich Afrika ausschließlich auf die Länder südlich der Sahara.

2 Freedom House klassifizierte 1989-1990 drei afrikanische Länder als frei, 11 Länder als teilweise frei und 33 Länder als unfrei; 2009 klassifizierte Freedom House 10 Länder als frei, 23 Länder als teilweise frei und lediglich 15 Länder als unfrei.

3 Der Transformationsprozess in den mittel- und osteuropäischen Nachbarstaaten stand und steht dagegen eindeutig stärker im Mittelpunkt des öffentlichen und des wissenschaftlichen Interesses.

4 Zwischen 1990 und 2003 fand in 44 von 49 afrikanischen Ländern mindestens eine Wahl statt. In 33 Staaten haben mindestens zwei aufeinanderfolgende Wahlen stattgefunden (Lynch/Crawford, 2010). 
Wahlen in der Elfenbeinküste, Kenia oder Simbabwe sind allseits geläufige Beispiele.

Der Demokratisierungsprozess hat somit vielerorts Rückschläge erfahren. Dies wirft die Frage auf, ob Wahlen von der Bevölkerung und den politischen Eliten als legitimes Mittel gesehen werden, eine Zuweisung von Macht, Kompetenzen und Ressourcen herzustellen, oder ob nicht alternative Verfahren dazu geeigneter wären. In diesem Beitrag argumentiere ich, dass Wahlen immer noch das legitimste Mittel sind, um in Afrika Macht zu verteilen. Dabei argumentiere ich auf Grundlage empirischer Forschungsergebnisse, nicht auf der Basis normativer oder philosophischer Überlegungen. Zunächst führe ich Argumentationslinien auf, die Wahlen in Afrika Legitimität absprechen oder ihre Legitimität zumindest bezweifeln. Im zweiten Teil verdeutliche ich anhand neuerlicher Befunde, dass Mehrparteienwahlen in weiten Teilen der afrikanischen Bevölkerung einen hohen Akzeptanzgrad genießen. Dies gilt gerade auch für die Staaten, in denen der Transitionsprozess zur Bildung defekter Demokratien geführt hat. Elektorale Gewalt kommt in Afrika wesentlich seltener vor, als es die öffentliche Berichterstattung suggeriert. Von einer generellen gewaltsamen Ablehnung von Wahlausgängen durch den Wahlverlierer kann nicht gesprochen werden. Schließlich gibt es heute in Afrika weder auf gesellschaftlicher noch auf politischer Ebene einen politischen Gegenentwurf, der eine ernstzunehmende intellektuelle Alternative zur Mehrparteiendemokratie darstellt.

\section{Wahlen als illegitimes Mittel für Machzuteilung}

Fundamentalkritische Stimmen halten Wahlen für grundsätzlich ungeeignet, um in Afrika ein Mindestmaß an politischer Legitimation für die Regierenden zu schaffen. Die (demokratischen) Institutionen des europäischen Nationalstaates sind eine westliche Errungenschaft und wurden den afrikanischen Staaten in der Kolonialzeit aufgezwungen. Wie alle europäischen Institutionen werden Wahlen von der afrikanischen Bevölkerung bestenfalls als fremd, schlimmstenfalls als Instrumente der Unterdrückung wahrgenommen. ${ }^{5}$ Per Definition kann von ihnen keine politische Legitimation ausgehen. Wo in Europa der Freiheit des Einzelnen kulturgeschichtlich eine hohe Bedeutung zugemessen wird, steht in der afrikanischen Kultur die Gruppe im Vordergrund. Genuin legitime staatliche Strukturen sollten sich an präkolonialen politischen Strukturen - wie eines Ältestenrates oder eines Rates von Stammesanführern - orientieren (Koelble/Lipuma 2008; für eine kohärente Zusammenfassung dieser und ähnlicher Argumente siehe Basedau 2003: 44-47).

5 Letzteres gilt für Länder, in denen keine demokratischen Institutionen geschaffen wurden. 
Ein weniger fundamentalkritischer Ansatz argumentiert, dass der politische Reformprozess den Forderungen der Bevölkerung Rechnung tragen muss, um ein politisches System zu legitimieren. Die Proteste der Zivilgesellschaft gegen die Machthaber zu Beginn der 1990er Jahre waren in erster Linie darauf ausgerichtet, eine weitere ökonomische Marginalisierung des Großteils der Bevölkerung zu verhindern, Armut nachhaltig zu bekämpfen und gerade der jüngeren Generation den sozialen Aufstieg zu ermöglichen. Sie hatten somit in erster Linie nicht das Ziel, prozedurale Reformen einzuleiten, sondern die soziale Situation der Bevölkerung zu verbessern. Afrikaner verbinden mit der Demokratisierung also die Hoffnung auf eine Befreiung von extremer Armut und nicht auf Mehrparteienwettstreit (Aké 1993). ${ }^{6}$ In der politikwissenschaftlichen Debatte über die politischen Transformationen dominieren jedoch Erhebungen von Messinstrumenten wie Freedom House oder der Bertelsmann Transformationsindex. Diese messen lediglich Fortschritte der prozeduralen Demokratie, treffen aber keine Aussage über Fortschritte bei der Armutsbekämpfung. Kurzum: Der afrikanische Kontinent hat einen Bedarf an sogenannter ,substantieller Demokratie“, weniger an prozeduraler Demokratie (Zuern 2009).

Ein dritter Argumentationsstrang drängt nicht so sehr auf Verbesserungen der substantiellen Demokratie, sondern sieht die Schwäche der prozeduralen Reformen vor allem darin, dass diese bislang nicht effektiv genug waren, politische Transparenz herzustellen und politischen Wandel zu ermöglichen. Wie angeführt gelten die meisten Regime in Afrika als defekte Demokratien bzw. als hybride Regime. Wahlen finden zwar statt, führen aber nicht zu einem Machtwechsel, sondern dienen lediglich dazu die herrschenden Eliten im Amt zu bestätigen. Reformorientierten Akteuren gelingt es nicht, die demokratischen Institutionen des Landes für ihren nominellen Zweck zu nutzen. Aufgrund ihres privilegierten Zugangs zu Ressourcen geling es Machthabern hingegen, demokratische Elemente zu kooptieren und zu schwächen (Gandhi 2008). In anderen Worten: Wahlen sind vielerorts eine reine Showveranstaltung.

Konfliktforscher sprechen Wahlen zwar nicht per se ihre Legitimität in Afrika ab, bezweifeln aber, dass sie von den politischen Eliten als legitimes Mittel zur Machtzuweisung akzeptiert werden. Sie warnen vor den negativen Konsequenzen von Parteienwettbewerb in multiethnischen Gesellschaften mit schwachen Institutionen. Demnach führt Mehrparteienwettbewerb zur Bildung ethnischer Parteien, wodurch

6 In der Literatur wird die Demokratisierungsbewegung der späten 1980er und frühen 1990er Jahre auch als ,second liberation“ bezeichnet. Die erste Befreiung steht für die Befreiung von kolonialer Unterdrückung. 
es zur Polarisierung kultureller Trennungslinien kommt. Demokratische Wahlen sind keine allgemein akzeptierten Abstimmungen über die Leistungsbilanz einer Regierung, sondern verkommen zu einer Mobilisierung ethnischer Gruppeninteressen. Die Anerkennung dieser Wahlen durch den Wahlverlierer ist unwahrscheinlich oder gar unmöglich. Von solchen Wahlen kann keine politische Legitimität für die Gesamtbevölkerung abgeleitet werden. Um diese zu erhalten, müsste der Wahlverlierer seine Niederlage eingestehen (Anderson u. a. 2005). Die starke ethnische Polarisierung, die von afrikanischen Parteien ausgeht, steht dem jedoch im Weg. Wahlen in Afrika schaffen somit keine politische Legitimität für den Wahlsieger; stattdessen münden sie in ethnischer Gewalt (Horowitz 2000).

\section{Legitimität von Mehrparteiendemokratie aus Sicht der Bevölkerung}

Die These, dass Mehrparteienwettbewerb für Afrika kulturell fremd ist, mag aus historischer Sicht zutreffen. Ob der Mehrparteienwettbewerb deswegen auch in der Gegenwart abgelehnt wird, ist damit nicht bewiesen. Neuere Umfrageerhebungen von Afrobarometer zeigen, dass demokratische Herrschaftsformen und somit auch Mehrparteienwettbewerb in weiten Teilen der afrikanischen Bevölkerung auf hohe Zustimmungsraten treffen. Die Zustimmungsrate zur Demokratie wird dabei mit der Zustimmungsrate zu alternativen Herrschaftssystemen kontrastiert. Ein Wunsch zu einer Rückkehr zu vorkolonialen Herrschaftsmodellen - die Herrschaft eines Stammesführers oder eines Ältestenrates - kann aus den vorliegenden Zahlen nicht herausgelesen werden. Tabelle 1 fasst ein paar prominente Ergebnisse zusammen.

Insgesamt genießt die Mehrparteiendemokratie eine hohe Zustimmung in der Bevölkerung. Augenscheinlich werden somit auch Wahlen als legitimes Mittel der Machzuteilung akzeptiert. Im Vergleich zu den jungen Demokratien Osteuropas und Lateinamerikas zeigen afrikanische Umfragen ein ähnliches hohes Maß an Unterstützung für die Mehrparteiendemokratie (Bratton/Mattes 2001 a). Zwischen einzelnen Ländern variiert die Unterstützung allerdings zu einem nicht unerheblichen Ausmaß. Im Jahr 2008 betrug sie beispielsweise in Madagaskar lediglich 39 Prozent und in Burkina Faso nur 46 Prozent; in Ghana dagegen betrug sie zum gleichen Zeitpunkt 78 Prozent und in Benin 81 Prozent. Botswana konnte mit 85 Prozent einen Spitzenwert verzeichnen. Diese Schwankungen implizieren, dass in den einzelnen Ländern des Kontinents der Demokratisierungsprozess von sehr unterschiedlichen politischen Dynamiken gekennzeichnet ist. Angesichts der Größe und der Heterogenität des Kontinents kann dies eigentlich nicht weiter überraschen. Bedauerlicherweise berücksichtigt die öffentliche Debatte diese Unterschiede höchst selten. 
Legitimität von Wahlen in der Transformation

Tabelle 1: Unterstützung der Demokratie durch afrikanische Bevölkerungen

\begin{tabular}{|l|c|c|c|c|c|}
\hline \multirow{2}{*}{$\begin{array}{l}\text { Welche Aussage } \\
\text { steht ihrer Meinung } \\
\text { am nächsten? }\end{array}$} & \multicolumn{5}{|c|}{ Afrobarometer Umfragedaten } \\
\cline { 2 - 6 } & $1999-2001$ & $2002-2003$ & $2005-2006$ & 2008 & $2011-2013$ \\
\hline $\begin{array}{l}\text { Bevorzugung der } \\
\text { Demokratie gegen- } \\
\text { über jeder anderen } \\
\text { Regierungsform }\end{array}$ & $69 \%$ & $64 \%$ & $62 \%$ & $70 \%$ & $79 \%$ \\
\hline $\begin{array}{l}\text { Ablehnung einer } \\
\text { Ein-Parteien- } \\
\text { Herrschaft }\end{array}$ & $69 \%$ & $67 \%$ & $71 \%$ & $73 \%$ & $77 \%$ \\
\hline $\begin{array}{l}\text { Ablehnung einer } \\
\text { Militärherrschaft }\end{array}$ & $82 \%$ & $77 \%$ & $73 \%$ & $75 \%$ & $83 \%$ \\
\hline $\begin{array}{l}\text { Ablehnung einer } \\
\text { Ein-Mann- } \\
\text { Herrschaft }\end{array}$ & $80 \%$ & $76 \%$ & $76 \%$ & $79 \%$ & $87 \%$ \\
\hline $\begin{array}{l}\text { Ablehnung der Ent- } \\
\text { scheidungsmacht ei- } \\
\text { nes Stammesführers } \\
\text { oder eines Ältesten- } \\
\text { rates }\end{array}$ & $69 \%$ & $53 \%$ & - & - & - \\
\hline
\end{tabular}

Quelle: http://www.afrobarometer.org

Detaillierte Analysen der Umfragedaten von Afrobarometer zeigen ferner, dass afrikanische Bürger unter Demokratie in erster Linie faire und freie Wahlen und bürgerliche Freiheitsrechte verstehen. Die Demokratie wird also mit bestimmten prozeduralen Vorgängen assoziiert - ähnlich wie in den Demokratien des Westens (Bratton/Mattes 2001 a). In Ländern, in denen Wahlen nicht demokratisch ablaufen, fordern afrikanische Bürger demokratischere Standards bei der Durchführung von Wahlen. Sie lehnen Wahlen und die Demokratie also nicht als illegitim ab, sondern fordern von ihren Eliten demokratische Standards einzuhalten. Selbiges trifft auch auf Länder zu, in denen Mehrparteienwahlen von Gewalt überschattet sind. Dies ist ein wichtiger Befund, zeigt er doch, dass aus der Existenz defekter Demokratien kein Legitimitätsdefizit demokratischer Normen und Institutionen abgeleitet werden kann (dies. 2001 b; Bratton 2010).

\section{Elektorale Gewalt: Eine systematische Bestandsaufnahme}

Mehrparteienwettstreit geht in vielen jungen multiethnischen Demokratien häufig mit Gewalt einher, was auf einen niedrigen Akzeptanzgrad von Wahlergebnissen hindeutet. Dies trifft leider auch auf Wahlen zu, die nach internationalen Standards 
als frei und fair gelten. Die jüngsten Wahlen in Kenia (2007/08), Simbabwe (2008), Nigeria (2011) oder der Elfenbeinküste (2011) bekräftigen die Befürchtung, dass politischer Wettbewerb in afrikanischen Gesellschaften zu bewaffneten Konflikten und Instabilität führt. Allerdings handelt es sich bei diesen Negativbeispielen um prominente Einzelfälle, nicht um generalisierbare Beobachtungen. Für jeden negativen Einzelfall könnte ein positives Beispiel genannt werden. Beispiele hierfür sind die Wahlen in Ghana (2008 und 2012), Benin (2006) und Senegal (2011) (Elischer 2009; Hartmann 2012; Stroh 2006). Bis vor Kurzem konzentrierte sich die empirische Forschung zu elektoraler Gewalt auch auf Einzelfallstudien oder auf Studien mit kleiner Fallzahl (Laakso 2007; Mehler 2007). Die Frage nach der generellen Häufigkeit von elektoraler Gewalt konnte lange Zeit empirisch nicht beantwortet werden.

Ein neuer Datensatz von Straus und Taylor (2012) analysiert die Intensität und Häufigkeit von elektoraler Gewalt in Afrika bei allen 221 Parlaments- und Präsidentschaftswahlen zwischen 1990 und 2008. Gewaltintensität wird dabei in vier Kategorien unterteilt:

- Kategorie 0: Wahlen ohne Gewalt;

- Kategorie 1: Wahlen, in denen es zur Androhung von Gewalt oder zu vereinzelten Zusammenstößen von Anhängern unterschiedlicher Parteien kommt;

- Kategorie 2: Wahlen mit gewaltsamer Repression über einen längeren Zeitraum hinweg;

- Kategorie 3: Wahlen mit 20 Todesopfern oder mehr.

Straus und Taylor finden schwere Vorfälle von Gewalt (Kategorie 3) in lediglich 10 Prozent aller afrikanischen Wahlen. In ebenfalls 10 Prozent der Fälle kommt es zu gewaltsamer Repression (Kategorie 2); in 38 Prozent der Fälle kommt es zu Gewalt mit geringen Auswirkungen (Kategorie 1). Schließlich sind 42 Prozent aller Wahlen von Gewalt überhaupt nicht betroffen. Über den Zeitraum von zwei Jahrzehnten ist diese Tendenz gleich geblieben, das heißt elektorale Gewalt nimmt über die Zeit weder zu noch ab. Afrikanische Wahlen sind somit sicherlich nicht gewaltfrei und werden in vielen Fällen von Teilen der Eliten (insbesondere von Wahlverlieren) nicht als legitim anerkannt. Jedoch führt Wählen nicht zwangsläufig zu (schwerer) Gewalt. Die große Mehrheit aller afrikanischen Wahlen verläuft gewaltfrei oder das Gewaltniveau ist deutlich geringer, als es der mediale Diskurs annehmen mag.

Diese erstmalig systematisch erhobenen Daten widersprechen der These, dass Partizipation und Wettbewerb in jungen Demokratien Stabilität und Frieden gefährden. Neuere Ergebnisse aus der afrikanischen Parteienforschung untermauern 
dies. Der Einfluss von Ethnizität auf afrikanisches Wahlverhalten und Parteiformationsprozesse ist wesentlich geringer als gemeinhin angenommen. Sowohl für afrikanische Wähler als auch für afrikanische Parteien sind ethnische Loyalitäten lediglich ein Faktor unter vielen, den es bei der Wahlentscheidung bzw. bei der Wahlkampfstrategie zu berücksichtigen gilt (Basedau u. a. 2011; Elischer 2013).

Eine genauere Analyse der Daten von Straus und Taylor ermöglicht eine Unterscheidung in Länder mit generell hoher Gewaltintensität, ${ }^{7}$ Länder, in denen es manchmal zu einem hohen Maß an Gewalt kommt, und Länder, die regelmäßig friedliche Wahlen erleben. ${ }^{8}$ Tabelle 2 führt diese Länder auf. Wie schon bei den Erhebungen von Afrobarometer zeigt sich, dass politischer Wettbewerb in Afrika trotz sehr ähnlicher Strukturbedingungen von unterschiedlichen Dynamiken gekennzeichnet ist.

Tabelle 2: Häufigkeit und Intensität von elektoraler Gewalt in Afrika

\begin{tabular}{|c|c|c|}
\hline $\begin{array}{l}\text { Länder mit durchweg friedlichen } \\
\text { Wahlen }\end{array}$ & $\begin{array}{l}\text { Länder, in denen es manchmal zu } \\
\text { starken Gewaltausbrüchen } \\
\text { kommt }\end{array}$ & $\begin{array}{l}\text { Länder, in denen es regelmäßig zu } \\
\text { starken Gewaltausbrüchen } \\
\text { kommt }\end{array}$ \\
\hline $\begin{array}{l}\text { Benin, Botswana, Burkina Faso, } \\
\text { Cape Verde, Chad, Djibouti, } \\
\text { Gabun, Gambia, Ghana, Guinea- } \\
\text { Bissau, Liberia, Malawi, Mauri- } \\
\text { tius, Mozambique, Namibia, Ru- } \\
\text { anda, Sao Tomé, Seychellen, Si- } \\
\text { erra Leone }\end{array}$ & $\begin{array}{l}\text { Angola, Burundi, Guinea, Kame- } \\
\text { run, Komoren, Lesotho, Mada- } \\
\text { gaskar, Mauretanien, Niger, Kon- } \\
\text { go, Republik Kongo, Senegal, } \\
\text { Tansania, Uganda, Zentralafrika- } \\
\text { nische Republik }\end{array}$ & $\begin{array}{l}\text { Äquatorialguinea, Äthiopien, } \\
\text { Demokratische Republik Kongo, } \\
\text { Elfenbeinküste, Kenia, Nigeria, } \\
\text { Südafrika, Sudan, Togo, Sim- } \\
\text { babwe }\end{array}$ \\
\hline
\end{tabular}

Quelle: Straus/Taylor 2009: 25.

\section{Wahlen in Afrika: Mehrheitlich legitim, politisch ohne Alternative}

Der Demokratisierungsprozess der letzten zwanzig Jahre bleibt in vielen Ländern Afrikas stark verbesserungsfähig. Gerade vor dem Hintergrund Afrikas widriger Strukturbedingungen liefern die hier ausgeführten Ergebnisse jedoch keinen Grund, die Legitimität afrikanischer Wahlen generell zu negieren. Auch wenn Afrikas politische Liberalisierung nicht zu einem Ende von wirtschaftlicher und sozialer Exklusion geführt hat, sehen weite Teile der afrikanischen Bevölkerung den Mehrparteienwettstreit als legitimes Mittel für die Allokation politischer Autorität - vor allem im Vergleich zu denjenigen (autokratischen) Herrschaftsformen, die in der Vergangenheit die afrikanische Politik bestimmten. Der Mangel an ,substantieller

7 Gemeint sind Länder, in denen es zu Gewalt der Kategorie 3 kommt.

8 Es liegt nicht nur Varianz zwischen den Ländern, sondern auch innerhalb der Länder vor. Auf diese kann in diesem Beitrag nicht eingegangen werden. 
Demokratie“ ist hierfür kein Hindernis. Eine gewünschte Rückkehr zu präkolonialen Herrschaftsformen wird ebenso abgelehnt. Der Mangel an „effektiver Demokratie" führt zu Forderungen nach qualitativ besseren, also genuin demokratischen Wahlen und somit zu mehr Demokratie. Die oftmals bemühte These, dass Wahlen in Afrika in Gewalt münden, lässt sich nicht bestätigen. Tatsächlich verlaufen Wahlen in der Mehrzahl der Staaten friedlich oder sind von einem geringen Gewaltniveau betroffen.

Schlussendlich sollte nicht ignoriert werden, dass weder auf gesellschaftlicher noch auf politischer Ebene ein intellektueller Gegenentwurf für eine legitimere Allokation von Macht existiert. Der öffentliche Diskurs Afrikas ist darauf ausgerichtet die Standards von Wahlen zu verbessern bzw. einmal erreichte Standards zu wahren. ${ }^{9}$ Transnationale afrikanische Zivilgesellschaften haben bereits seit Jahren unterschiedliche Vorschläge unterbreitet, wie die Qualität von Wahlen weiter verbessert werden kann (siehe beispielsweise EISA 2010). Auf politischer Ebene hat die Afrikanische Union kürzlich die „African Charter on Democracy, Elections and Governance" ratifiziert, in der Wahlen als einziges legitimes Mittel angesehen werden, Macht zu verteilen. Die Vorbereitung der militärischen Intervention der westafrikanischen Regionalorganisation ECOWAS in Mali verfolgt nicht nur das geostrategische Interesse, den malischen Staat aus den Händen islamistischer Gruppen zu befreien, sondern eine demokratische legitimierte Regierung einzusetzen. Natürlich werden diese ehrgeizigen Ziele nicht immer erreicht. In vielen Fällen versuchen die politischen Eliten, sie aktiv zu unterwandern. Wer die Legitimität von afrikanischen Wahlen abstreitet, muss jedoch letztlich über alternative Methoden der Herrschaftslegitimierung nachdenken. Trotz aller Rückschläge, die Afrikas Transitionsversuche auszeichnen, sind solche Alternativen bislang nicht sichtbar.

\section{Literatur}

Aké, Claude, 1993: The Unique Case of African Democracy, in: International Affairs 69, 239-244.

Anderson, Christopher/Blais, André/Bowler, Shaun/Donovan, Todd/Listhaug, Ola, 2005: Losers' Consent: Elections and Democratic Legitimacy, Oxford.

9 Dies bezieht sich auf die persönliche Erfahrung des Autors während unterschiedlichster Feldforschungsaufenthalte in insgesamt zehn afrikanischen Ländern. Mögliche Gegenentwürfe beinhalten auch die Möglichkeit einer Abkehr vom laizistischen Staatswesen und die Herrschaft von religiösen Führern wie dies beispielsweise in den Staaten Nordafrikas auf gesellschaftlicher und politischer Ebene diskutiert wird. 
Basedau, Matthias, 2003: Erfolgsbedingungen von Demokratie im subsaharischen Afrika, Opladen.

Basedau, Matthias/Erdmann, Gero/Lay, Jann/Stroh, Alexander, 2011: Ethnicity and Party Preference in sub-Saharan Africa, in: Democratization 18 (2), 462-489.

Bratton, Michael, 2010: Anchoring the "D-Word" in Africa, in: Journal of Democracy 21 (4), 106-113.

Bratton, Michael/Mattes, Robert, 2001 a: How People View Deocracy. Africans Surprising Universalism, in: Journal of Democracy 12 (1), 107-121.

Bratton, Michael/Mattes, Robert, 2001 b: Support for Democracy in Africa: Intrinsic or Instrumental?, in: British Journal of Political Science 31 (3), 447-474.

Electoral Institute for the Sustainability of Democracy in Africa (EISA), 2010: When

Elections Become a Curse. Redressing Electoral Violence in Africa, Johannesburg.

Elischer, Sebastian, 2008: Do African Parties Contribute to Democracy? Some Findings from Kenya, Ghana and Nigeria, in: Afrika Spectrum 43 (2), 175-201. Elischer, Sebastian, 2009: Afrikas neues Vorbild? Ghana auf dem Weg der demokratischen Konsolidierung. GIGA Focus Afrika 01/2009, Hamburg.

Elischer, Sebastian, 2013: Political Parties in Africa. Ethnicity and Party Formation, Cambridge (i. E.).

Erdmann, Gero, 2002: Neopatrimoniale Herrschaft - oder: Warum es in Afrika so viele Hybridregime gibt, in: Petra Bendel/Aurel Croissant/Friedbert Rüb (Hrsg.), Zwischen Demokratie und Diktatur: Zur Konzeption und Empirie demokratischer Grauzonen, Opladen, 323-341.

Gandhi, Jennifer, 2008: Political Institutions under Dictatorship, Cambridge.

Hartmann, Christof, 2012: Machtwechsel im Senegal - neue Chance für die Demokratie? GIGA Focus Afrika 2/2012, Hamburg.

Horowitz, Donald, 2000: Ethnic Groups in Conflict, Berkeley.

Huntington, Samuel, 1991: The Third Wave, Oklahoma.

Joseph, Richard, 1991: Africa: The Rebirth of Political Freedom, in: Journal of Democracy 2 (4), 11-24.

Koelble, Thomas/Lipuma, Edward, 2008: Democratizing Democracy: A Postcolonial Critique of Conventional Approaches to the "Measurement of Democracy", in: Democratization 15 (1), 1-28.

Laakso, Liisa, 2007: Insights into Electoral Violence, in: Matthias Basedau/Gero Erdmann/Andreas Mehler (Hrsg.), Votes, Money and Violence. Political Parties and Elections in Africa, Nordiska Afrikainstitutet/University of KwaZulu-Natal Press, 224-252. 
Levitsky, Steven/Way, Lucas, 2011: Competitive Authoritarianism. International Linkages, Organizational Power, and the Fate of Hybrid Regimes, Cambridge/ New York.

Lynch, Gabrielle/Crawford, Gordon, 2010: Democratization in Africa 1990-2010: An Assessment, in: Democratization 18 (2), 275-310.

McFerson, Hazel, 1992: Democracy and Development in Africa, in: Journal of Peace Research 29, 241-248.

Mehler, Andreas, 2007: Political Parties and Violence in Africa: Systematic Reflections against Empirical Background, in: Matthias Basedau/Gero Erdmann/ Andreas Mehler (Hrsg.), Votes, Money and Violence. Political Parties and Elections in Africa, Nordiska Afrikainstitutet/University of KwaZulu-Natal Press, 194-223.

Merkel, Wolfgang/Puhle, Hans-Jürgen/Croissant, Aurel/Eicher, Claudia/Thiery, Peter, 2003: Defekte Demokratie. Band 1: Theorie, Opladen.

Straus, Scott/Taylor, Charlie, 2009: Democratization and Electoral Violence in SubSaharan Africa 1990-2007, American Association of Political Science 2009.

Stroh, Alexander, 2006: Kaurimuschel statt Chamäleon: Dritter demokratischer Präsidentenwechsel in Benin, GIGA Focus 08/2006, Hamburg.

Zuern, Elke, 2009: Democratization as Liberation: Competing African Perspectives on Democracy, in: Democratization 16 (3), 585-603.

Korrespondenzanschrift:

Prof. Dr. Sebastian Elischer

Leuphana Universität Lüneburg

Scharnhorststr. 1, C12.308

21335 Lüneburg

E-Mail: elischer@leuphana.de

German Institute of Global and Area Studies

Neuer Jungfernstieg 21

20354 Hamburg

E-Mail: sebastian.elischer@giga-hamburg.de 